\title{
FactorNet: a deep learning framework for predicting cell type specific transcription factor binding from nucleotide-resolution sequential data
} Daniel Quang ${ }^{1,2}, X_{1}$ iaohui Xie ${ }^{1,2, *}$

\author{
1 Department of Computer Science, University of California, Irvine, CA 92697, USA \\ 2 Center for Complex Biological Systems, University of California, Irvine, CA 92697, USA
}

\section{*xhx@ics.uci.edu}

Key words: deep learning, transcription factors, convolutional neural network, recurrent neural network

\section{Abstract}

Due to the large numbers of transcription factors (TFs) and cell types, querying binding profiles of all $\mathrm{TF} /$ cell type pairs is not experimentally feasible, owing to constraints in time and resources. To address this issue, we developed a convolutional-recurrent neural network model, called FactorNet, to computationally impute the missing binding data. FactorNet trains on binding data from reference cell types to make accurate predictions on testing cell types by leveraging a variety of features, including genomic sequences, genome annotations, gene expression, and single-nucleotide resolution sequential signals, such as DNase I cleavage. To the best of our knowledge, this is the first deep learning method to study the rules governing TF binding at such a fine resolution. With FactorNet, a researcher can perform a single sequencing assay, such as DNase-seq, on a cell type and computationally impute dozens of TF binding profiles. This is an integral step for reconstructing the complex networks underlying gene regulation. While neural networks can be computationally expensive to train, we introduce several novel strategies to significantly reduce the overhead. By visualizing the neural network models, we can interpret how the model predicts binding which in turn reveals additional insights into regulatory grammar. We also investigate the variables that affect cross-cell type predictive performance to explain why the model performs better on some TF/cell types than others, and offer insights to improve upon this field. Our method ranked among the top four teams in the ENCODE-DREAM in vivo Transcription Factor Binding Site Prediction Challenge. 


\section{Introduction}

High-throughput sequencing has led to a diverse set of methods to interrogate the epigenetic landscape for the purpose of discovering tissue and cell type-specific putative functional elements. Such information provides valuable insights for a number of biological fields, including synthetic biology and translational medicine. Among these methods are ChIP-seq, which applies a large-scale chromatin immunoprecipitation assay that maps in vivo transcription factor (TF) binding sites or histone modifications genome-wide (Johnson et al. 2007), and DNase-seq, which identifies genome-wide locations of open chromatin, or "hotspots", by sequencing genomic regions sensitive to DNase I cleavage (Crawford, G. et al., 2006, John et al., 2013). At deep sequencing depth, DNase-seq can identify TF binding sites, which manifest as dips, or "footprints", in the digital DNase I cleavage signal (Hesselberth et al., 2009; Boyle et al., 2011; Neph, S. et al. 2012). Other studies have shown that cell type-specific functional elements can display unique patterns of motif densities and epigenetic signals (Quang et al. 2015b). Computational methods can integrate these diverse datasets to elucidate the complex and non-linear combinations of epigenetic markers and raw sequence contexts that underlie functional elements such as enhancers, promoters, and insulators. Some algorithms accomplish this by dividing the entire genome systematically into segments, and then assigning the resulting genome segments into "chromatin states" by applying machine learning methods such as Hidden Markov Models, Dynamic Bayesian Networks, or Self-Organizing Maps (Ernst and Kellis, 2012, Hoffman et al., 2012, Mortazavi et al., 2013).

The Encyclopedia of DNA Elements (ENCODE) (ENCODE Project Consortium, 2012) and NIH Roadmap Epigenomics (Roadmap Epigenomics Consortium et al. 2015) projects have generated a large number of ChIP-seq and DNase-seq datasets for dozens of different cell and tissue types. Owing to several constraints, including cost, time or sample material availability, these projects are far from completely mapping every mark and sample combination. This disparity is especially large for TF binding profiles because ENCODE has profiled over 600 human biosamples and over 200 TFs, translating to over 120,000 possible pairs of biosamples and TFs, but as of the writing of this article only about 8,000 TF binding profiles are available. Due to the strong correlations between epigenetic markers, computational methods have been proposed to impute the missing datasets. One such imputation method is ChromImpute Ernst 27 and Kellis, 2015), which applies ensembles of regression trees to impute missing chromatin marks. With the ${ }_{28}$ exception of CTCF, ChromImpute does not impute TF binding. Moreover, ChromImpute does not take 29 sequence context into account, which can be useful for predicting the binding sites of TFs like CTCF that 30 are known to have a strong binding motif. 
Computational methods designed to predict TF binding include PIQ (Sherwood et al., 2014), Centipede 32 (Pique-Regi et al., 2011), and msCentipede (Raj et al., 2015). These methods require a collection of motifs 33 and DNase-seq data to predict TF binding sites in a single tissue or cell type. While such an approach can 34 be convenient because the DNase-seq signal for the cell type considered is the only mandatory experimental 35 data, it has several drawbacks. These models are trained in an unsupervised fashion using algorithms such as 36 expectation maximization (EM). From our experience, EM-based algorithms can be very computationally ${ }_{37}$ inefficient. To compensate for this issue, PIQ, Centipede, and msCentipede limit training and evaluation to 38 motif matches, which represent a small and unrepresentative fraction of the whole genome. Furthermore, the 39 manual assignment of a motif for each TF is a strong assumption that completely ignores any additional 40 sequence contexts such as co-binding, indirect binding, and non-canonical motifs. This can be especially ${ }^{41}$ problematic for TFs like REST, which is known to have eight non-canonical binding motifs Quang and Xie, ${ }_{42}$ 2014).

More recently, deep neural network (DNN) methods have gained significant traction in the bioinformatics community. DNNs are useful for biological applications because they can efficiently identify complex non-linear patterns from large amounts of feature-rich data. They have been successfully applied to predicting splicing patterns (Leung et al. 2014), predicting variant deleteriousness (Quang et al., 2015a), and gene expression inference (Chen et al. 2016). The convolutional neural network (CNN), a variant of the DNN, has been useful for genomics because it can process raw DNA sequences and the kernels are analogues to position weight matrices (PWMs), which are popular models for describing the sequence-specific binding pattern of TFs. Examples of genomic application of CNNs include DanQ(Quang and Xie, 2016), DeepSEA (Zhou and Troyanskaya, 2015), Basset (Kelley et al., 2016), DeepBind (Alipanahi et al., 2015), and DeeperBind (Hassanzadeh and Wang, 2016). These methods accept raw DNA sequence inputs and are trained in a supervised fashion to discriminate between the presence and absence of epigenetic markers, including TF binding, open chromatin, and histone modifications. Consequently, these algorithms are not suited to the task of predicting epigenetic markers across cell types. Instead, they are typically designed for other tasks such as motif discovery or functional variant annotation. Both DanQ and DeeperBind, unlike the other three CNN methods, also use a recurrent neural network (RNN), another type of DNN, to form a CNN-RNN hybrid architecture that can outperform pure convolutional models. RNNs have been useful in pther machine learning applications involving sequential data, including phoneme classification Graves and Schmidhuber, 2005), speech recognition (Graves et al. 2013), machine translation (Sundermeyer et al., 2014), 61 and human action recognition (Zhu et al. 2016). More recently, CNNs and RNNs have been used for predicting single-cell DNA methylation states (Angermueller et al., 2017). 


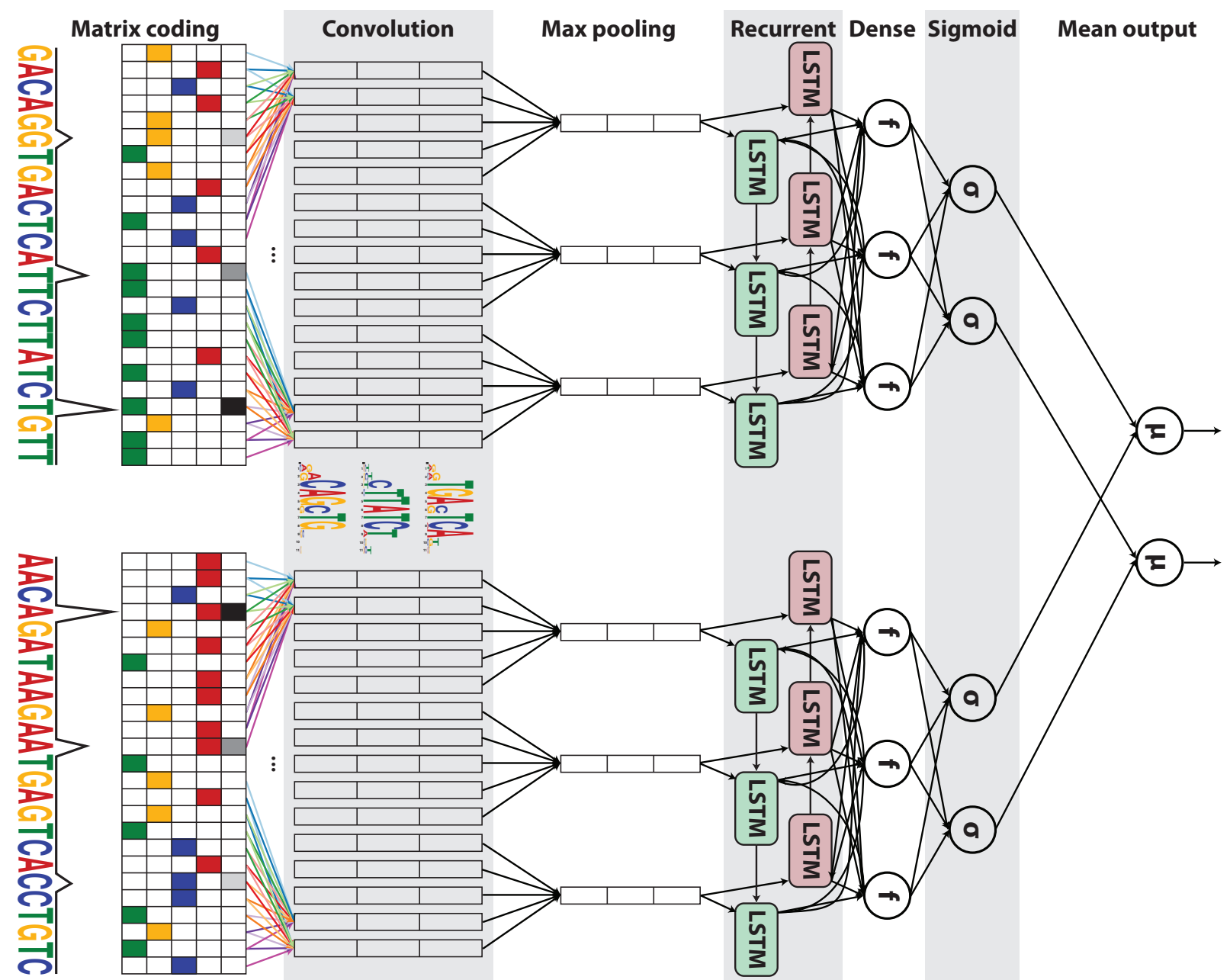

Figure 1. Simplified diagram of the FactorNet model. An input DNA sequence (top) is first one hot encoded into a 4-row bit matrix. Real-valued single-nucleotide signal values are concatenated as extra rows to this matrix. A rectifier activation convolution layer transforms the input matrix into an output matrix with a row for each convolution kernel and a column for each position in the input (minus the width of the kernel). Each kernel is effectively a sequence motif. Max pooling downsamples the output matrix along the spatial axis, preserving the number of channels. The subsequent recurrent layer contains long short term memory (LSTM) units connected end-to-end in both directions to capture spatial dependencies between motifs. Recurrent outputs are densely connected to a layer of rectified linear units. The activations are likewise densely connected to a sigmoid layer that nonlinear transformation to yield a vector of probability predictions of the TF binding calls. An identical network, sharing the same weights, is also applied to the reverse complement of the sequence (bottom). Finally, respective predictions from the forward and reverse complement sequences are averaged together, and these averaged predictions are compared via a loss function to the true target vector. Although not pictured, we also include a sequence distributed dense layer between the convolution and max pooling layer to capture higher order motifs. 
To predict cell type-specific TF binding, we developed FactorNet, which combines elements of the aforementioned algorithms. FactorNet trains a DNN on data from one or more reference cell types for which the TF or TFs of interest have been profiled, and this model can then predict binding in other cell types. The FactorNet model builds upon the DanQ CNN-RNN hybrid architecture by including additional real-valued coordinated-based signals such as DNase-seq signals as features. FactorNet is similar to a recently developed method called DeepCpG, which integrates sequence context and neighboring methylation rates to predict single-cell DNA methylation states using a CNN and a bidirectional RNN (Angermueller et al., 2017). We also extended the DanQ network into a "Siamese" architecture that accounts for reverse complements (Figure 11. This Siamese architecture applies identical networks to both strands to ensure that both the forward and reverse complement sequences return the same outputs, essentially halving the total amount of training data, ultimately improving training efficiency and predictive accuracy. Both networks share the same weights. Siamese networks are popular among tasks that involve finding similarity or a relationship between two comparable objects. Two examples are signature verification (Bromley et al., 1993) and assessing sentence similarity (Mueller and Thyagarajan, 2016). Another recent method, TFImpute (Qin and Feng, 2017), shares many similarities with FactorNet. Like FactorNet, TFImpute is intended to impute missing TF binding

We submitted the FactorNet model to the ENCODE-DREAM in vivo Transcription Factor Binding Site Prediction Challenge (https://www.synapse.org/ENCODE), where it placed among the top four ranked teams. All results discussed in this paper are derived from data in the Challenge. The Challenge delivers a 84 crowdsourcing approach to figure out the optimal strategies for solving the problem of TF binding prediction.

\section{Results}

\section{Predictive performance varies across transcription factors}

Table 1 shows a partial summary of FactorNet cross-cell type predictive performances on a variety of cell type and TF combinations as of the conclusion of the ENCODE-DREAM Challenge. Final rankings in the Challenge are based on performances over $13 \mathrm{TF} /$ cell type pairs. A score combining several primary performance measures is computed for each pair. In addition to the $13 \mathrm{TF} /$ cell type pairs for final rankings, there are $28 \mathrm{TF} /$ cell type "leaderboard" pairs. Competitors can compare performances and receive live 
Table 1. Partial summary of FactorNet cross-cell type predictive performances on the ENCODE-DREAM Challenge data. Each final ranking TF/cell type pair is demarcated with a *. For each final ranking TF/cell type pair, we provide, in parentheses, performance scores based on the evaluation pair's original ChIP-seq fold change signal.

\begin{tabular}{lllll} 
& & & & Recall at \\
Factor & Cell type & auROC & auPR & 50\% FDR \\
\hline CTCF & iPSC & $0.9966(0.9998)$ & $0.8608(0.9794)$ & $0.9142(0.9941)$ \\
CTCF & GM12878 & 0.9968 & 0.8451 & 0.8777 \\
CTCF* & PC-3 & $0.9862(0.9942)$ & $0.7827(0.8893)$ & $0.7948(0.9272)$ \\
ZNF143 & K562 & 0.9884 & 0.6957 & 0.7303 \\
MAX & MCF-7 & 0.9956 & 0.6624 & 0.8290 \\
MAX* & liver & $0.9882(0.9732)$ & $0.4222(0.6045)$ & $0.3706(0.6253)$ \\
EGR1 & K562 & 0.9937 & 0.6522 & 0.7312 \\
EGR1* & liver & $0.9856(0.9741)$ & $0.3172(0.5306)$ & $0.2164(0.5257)$ \\
\hline HNF4A & liver & $0.9785(0.9956)$ & $0.6188(0.8781)$ & $0.6467(0.9291)$ \\
MAFK & K562 & 0.9946 & 0.6176 & 0.6710 \\
MAFK & MCF-7 & 0.9906 & 0.5241 & 0.5391 \\
GABPA & K562 & 0.9957 & 0.6125 & 0.6299 \\
GABPA* & liver & $0.9860(0.9581)$ & $0.4416(0.5197)$ & $0.3550(0.5202)$ \\
YY1 & K562 & 0.9945 & 0.6078 & 0.7393 \\
TAF1 & HepG2 & 0.9930 & 0.5956 & 0.6961 \\
TAF1* & liver & $0.9892(0.9657)$ & $0.4283(0.4795)$ & $0.4039(0.4766)$ \\
E2F6 & K562 & 0.9885 & 0.5619 & 0.6455 \\
REST & K562 & 0.9958 & 0.5239 & 0.5748 \\
REST* & liver & $0.9800(0.9692)$ & $0.4122(0.5596)$ & $0.4065(0.5945)$ \\
FOXA1* & liver & $0.9862(0.9813)$ & $0.4922(0.6546)$ & $0.4889(0.6728)$ \\
FOXA1 & MCF-7 & 0.9638 & 0.4487 & 0.4613 \\
\hline JUND & H1-hESC & 0.9948 & 0.4098 & 0.3141 \\
JUND* & liver & $0.9765(0.9825)$ & $0.2649(0.6921)$ & $0.1719(0.7223)$ \\
TCF12 & K562 & 0.9801 & 0.3901 & 0.3487 \\
STAT3 GM12878 & 0.9975 & 0.3774 & 0.3074 \\
NANOG* & iPSC & $0.9885(0.9876)$ & $0.3539(0.6421)$ & $0.3118(0.6680)$ \\
CREB1 & MCF-7 & 0.9281 & 0.3105 & 0.2990 \\
E2F1* & K562 & $0.9574(0.9888)$ & $0.2406(0.6428)$ & $0.0000(0.6573)$ \\
\hline FOXA2* & liver & $0.9773(0.9932)$ & $0.2172(0.7920)$ & $0.0231(0.8278)$ \\
& & & &
\end{tabular}

updating of their scores for the leaderboard TF/cell type pairs. Scores for the 13 final ranking TF/cell type pairs were not available until the conclusion of the challenge. Our model achieved first place on six of the 13 $\mathrm{TF} /$ cell type final ranking pairs, the most of any team.

FactorNet typically achieves auROC scores above $97 \%$ for most of the $\mathrm{TF} /$ cell type pairs, reaching as low as $92.8 \%$ for CREB1/MCF-7. auPR scores, in contrast, display a wider range of values, reaching as low as $21.7 \%$ for FOXA1/liver and $87.8 \%$ for CTCF/iPSC. For some TFs, such as CTCF and ZNF143, the predictions are already accurate enough to be considered useful. Much of the variation in auPR scores can be attributed to noise in the ChIP-seq signal used to generate the evaluation labels, which we demonstrate by building classifiers based on taking the mean in a $200 \mathrm{bp}$ window of the ChIP-seq fold change signal with 10 respect to input control. Peak calls are derived from the SPP algorithm (Kharchenko et al. 2008), which 102 
uses the fold-change signal and peak shape to score and rank peaks. An additional processing step scores peaks according to an irreproducible discovery rate (IDR), which is a measure of consistency between

We can also visualize the FactorNet predictions as genomic signals that can be viewed alongside the peak regions.

The ENCODE-DREAM challenge data, documentation, and results can be found on the Challenge

\section{Interpreting neural network models}




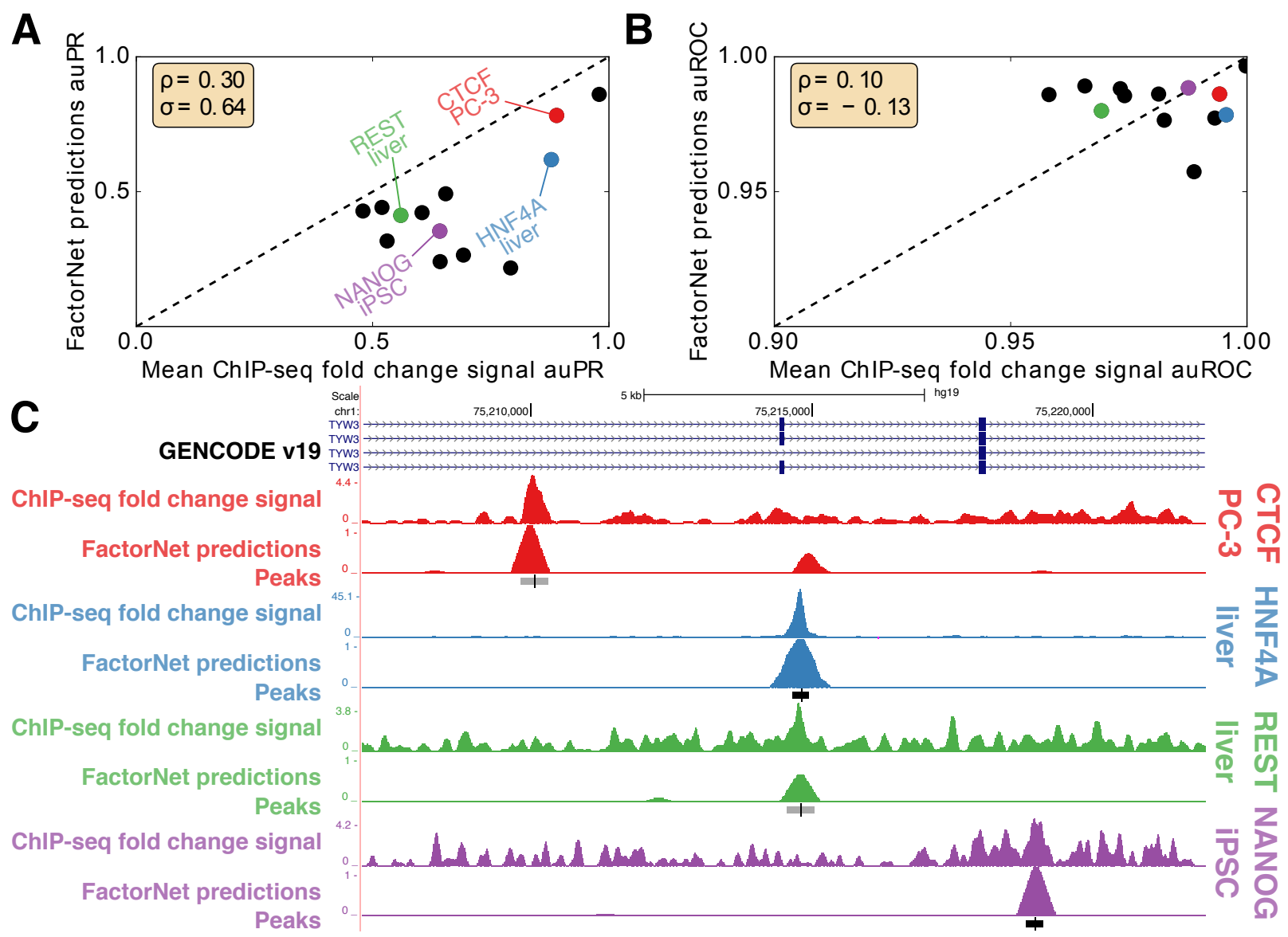

Figure 2. Predictive performance and ChIP-seq signal varies across TF/cell-type pairs. Scatterplots compare $(A)$ auPR and $(B)$ auROC scores between FactorNet predictions and mean ChIP-seq fold change signal. Each marker corresponds to one of the 13 final ranking TF/cell type pairs. Spearman $(\rho)$ and Pearson $(\sigma)$ correlations are displayed in each plot. (C) Genome browser (Kent et al. 2002) screenshot displays the ChIP-seq fold change signal, FactorNet predictions, and peak calls for four TF/cell type pairs in the TYW3 locus. Confidently bound regions are more heavily shaded than ambiguously bound regions.

2013). To generate a saliency map, we compute the gradient of the output category with respect to the input single-nucleotide resolution. Using a liver HNF4A peak sequence and HNF4A predictor model as an example, ${ }_{136}$ the saliency map highlights a subsequence overlapping the summit that strongly matches the known ${ }_{137}$ canonical HNF4A motif, as well as two putative binding sites upstream of the summit on the reverse ${ }_{138}$ complement (Figure 3D). 
A

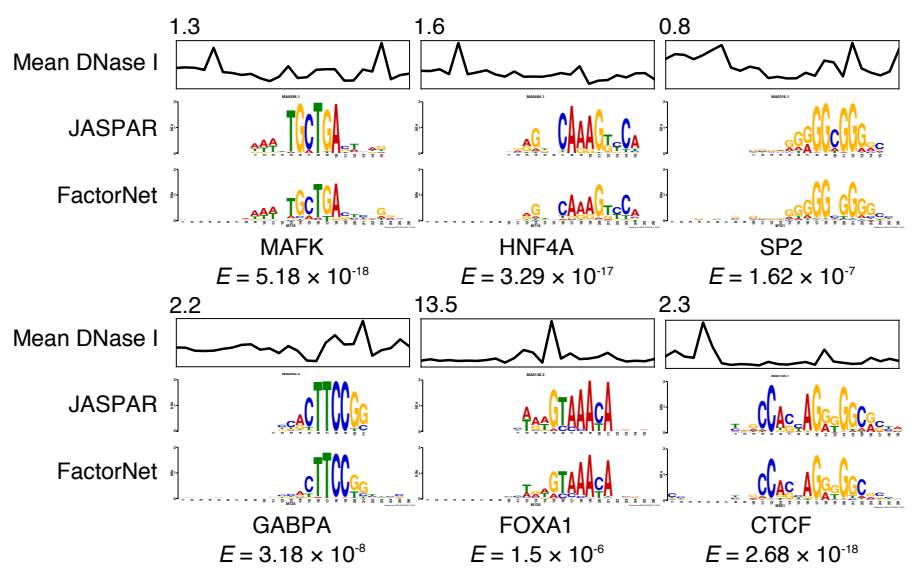

B

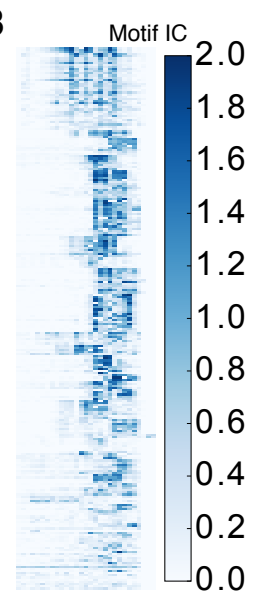

C

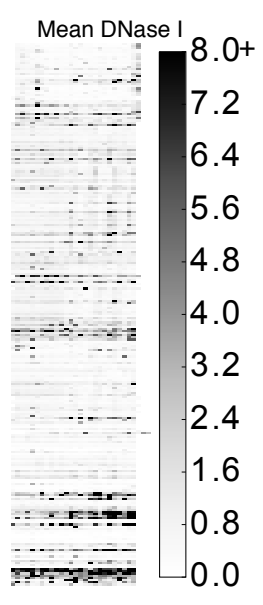

D
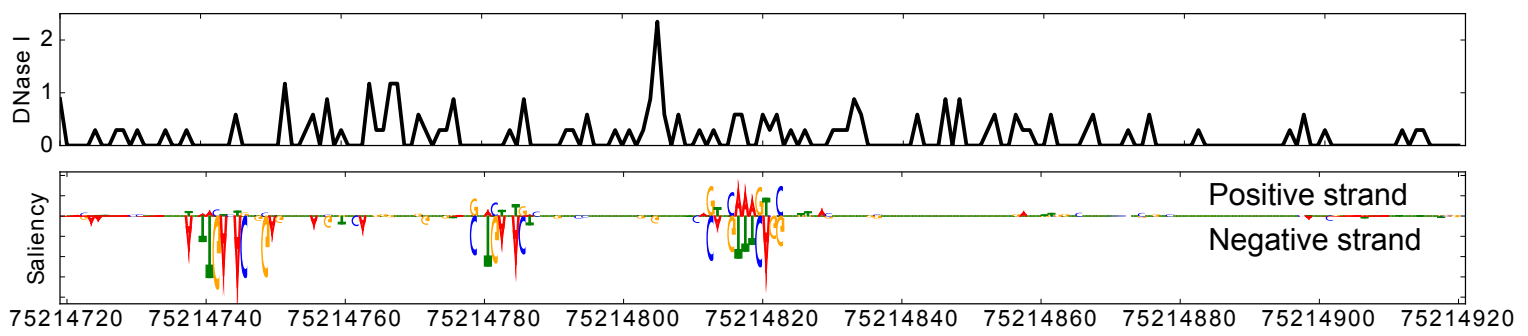

Figure 3. Visually interpreting FactorNet models. (A) Network kernels from a HepG2 multi-task FactorNet model are converted to sequence logos and aligned with motifs from JASPAR (Mathelier, A. et al., 2016) using TOMTOM (Gupta et al. 2007). Mean normalized DNase I cleavage signals and their maximum values are displayed above the aligned logos. $E$-values measure similarity between query and target motifs, corrected for multiple hypothesis testing. All kernels are converted to sequence logos and aligned with RSAT (Medina-Rivera et al. 2015). The heatmaps are ordered by this alignment and colored according to the motif information content (IC) (B) or mean DNase I cleavage signal $(C)$ at each nucleotide position. $(D)$ Normalized liver DNase I cleavage signal and saliency maps of aligned stranded sequences centered on the summit of a liver HNF4A peak in the TYW3 locus (Figure 2C). Negative gradients are converted to zeros. We visualized saliency maps with the DeepLIFT visualizer (Shrikumar et al., 2017)

\section{Data variation influences predictive performance}

In the cases for which two or more testing cell types are available for the same TF, we also observe some

rather large disparities in performance. With the exception of FOXA1, FactorNet consistently performs poorer for liver than for other cell types, the difference in auPR reaching as much as $33.5 \%$ in the case of EGR1 (Table 1). Variation in data quality across cell type-specific datasets may partially explain these performance differences. The DNase-seq data, which is arguably the most informative cell type-specific 
To further scrutinize the effect data variation has on performance, we trained several FactorNet single-task models and plotted the learning curves to monitor for overfitting (Figure $\mathrm{S} 2 \mathrm{~B}$ ). Learning curves trace the predictive performance of neural networks on training and validation sets. They are useful for ${ }_{152}$ identifying signs of overfitting, a common problem in machine learning. These learning curves focus on the ${ }_{153}$ GM12878 and HeLa-S3 cell types, using one cell type for training and the other as a validation set. We ${ }^{154}$ selected these two cell types because they are the only two reference cell types for E2F1, which FactorNet ${ }_{155}$ performed particularly poor on. In addition, the HeLa-S3 DNase-seq data read count and FRiP score are ${ }_{156}$ both almost twice that of the read count and FRiP score for the GM12878 DNase-seq data.

From the learning curves of the E2F1 model trained on GM12878, we observe evidence of overfitting. The HeLa-S3 cross-cell type validation loss reaches a minimum value within four training epochs, after which it ${ }^{159}$ increases until it reaches a steady state value. In contrast, the GM12878 within-cell type validation loss steadily decreases past the first four epochs and remains much smaller than the HeLa-S3 validation loss throughout training. At first, we speculated the gap to be caused by the differences in the cell type DNase-seq data; however, based on the learning curves for other TFs, this may not necessarily be the sole reason. In the cases of GABPA and TAF1, the differences in validation losses is much smaller. One possible explanation for these results is the differences in the ChIP-seq protocols between the GM12878 and HeLa-S2 datasets. Unlike the other three TFs, the GM12878 and HeLa-S3 E2F1 ChIP-seq datasets were generated using two different antibodies: ENCAB037OHX and ENCAB000AFU, respectively. Both ZNF143 ChIP-seq datasets were generated using the same antibody (ENCAB000AMR), but the model trained on HeLa-S3 displays an unusually high validation loss difference. We speculate this is because the GM12878 ZNF143 169 ChIP-seq dataset was generated using both single-end $36 \mathrm{bp}$ and paired-end $100 \mathrm{bp}$ reads while the HeLa-S3 ${ }_{170}$ ZNF143 ChIP-seq dataset was generated using only single-end 36 bp reads. Given that paired-end 100 bp ${ }_{171}$ reads can map to genomic regions that are unmapable for the shorter 36 bp reads, we suspect that $\quad{ }_{172}$ differences in read types can introduce significant dataset-specific artifacts.

Given the differences in the GM12878 and HeLa-S3 E2F1 ChiP-seq datasets resulting from the use of ${ }_{174}$ different antibodies, we investigated whether a model exclusively trained on one cell type could improve our $\quad 175$ predictive performance for the K562/E2F1 testing set. To do so, we retrained single- and multi-task models ${ }_{176}$ exclusively on either GM12878 or HeLa-S3 and evaluated cross-cell type binding performance on the ${ }_{177}$ E2F1/K562 testing set. In contrast, the E2F1 model used at the conclusion of the Challenge was trained on ${ }_{178}$ data from both reference cell types. The K562 E2F1 ChIP-seq dataset was generated using the antibodies ${ }_{179}$ ENCAB037OHX and ENCAB851KCY, the former of which was also used for GM12878. Hence, we expect ${ }_{180}$ that the GM12878 model would be a better predictor for K562 E2F1 binding sites than the other two models, ${ }_{181}$ 
which we find to indeed be the case (Figure S2 C-D). Although we managed to improve upon our previous E2F1 model, the cross-cell type performance for E2F1 is still inadequate, especially compared to TFs like

\section{Comparing single- and multi-task training}

Although a thorough comparison between single- and multi-task training is beyond the scope of this paper,

While we initially assumed that the multi-task training confers an advantage by introducing additional bins from multiple ChIP-seq datasets. By increasing the ratio of negative to positive samples per epoch for ${ }_{201}$ single-task training, we can close the gap between the two training methods in terms of the auPR score, 202 demonstrating that the selection of negative bins affects predictive accuracy. Moreover, the single-task models each use 654,657 weights and require 30 seconds- 5 minutes per training epoch whereas the multi-task models each use 5.4 million weights and require 2-3 hours per training epoch, making the former significantly 


\section{Discussion}

In this work, we introduced FactorNet - an open source package to apply stacked convolutional and recurrent 211 neural networks for predicting TF binding across cell types. While RNNs are computationally expensive to ${ }^{212}$ train, especially compared to CNNs, FactorNet incorporates several heuristics to significantly speed up model 213 training and improve predictive performance. Using data from the ENCODE-DREAM Challenge, we ${ }^{214}$ demonstrated how our model can effectively integrate cell type-specific data such as DNase-seq to generalize 215 TF binding from reference cell types to testing cell types. As of the conclusion of the Challenge, FactorNet is ${ }^{216}$ one of the top performing binding prediction models.

Through our post-Challenge analyses, we gained insights into the variables that affect predictive leveraging the original ChIP-seq fold change signal, we established upper bounds for the auPR metric for each final ranking $\mathrm{TF} /$ cell type pair. These bounds also correlate positively with auPR scores from FactorNet predictions, showing that a large amount of the variation in predictive performance can be attributed to the noise in the original ChIP-seq signal (Figure 2A). We expect that predictive performance for many $\mathrm{TF} /$ cell type pairs can be improved by redoing experiments with higher quality antibodies. non-protein-bound DNA fragments (Rhee and Pugh, 2011), may improve the quality of ChIP signals. Next, we investigated the variation in the DNase-seq datasets. We found that the DNase-seq datasets greatly differ in terms of sequencing depth and SNR (Figure S3). While we do correct for the variation in sequencing depth by normalizing the cleavage signals to $1 \mathrm{x}$ coverage, we do not correct for the variation in the SNR. The performance lost is most staggering for the liver cell type, which has the DNase-seq dataset with the lowest ${ }^{231}$ SNR. However, differences in DNase-seq SNR do not fully account for differences in predictive performance. ${ }^{232}$ By studying several within- and cross-cell type validation curves, we also concluded that differences in antibodies and read lengths can introduce significant dataset-specific biases (Figure S2B). Accordingly, we can improve performance by omitting less compatible cell type datasets (Figure S2 C-D).

We also compared single- and multi-task training frameworks. Several deep learning methods, including 
testing set, the GM12878 single-task model outperformed GM12878 multi-task model (Figure S2C); however, 241 for the NANOG/iPSC cross-cell type testing set, the H1-hESC multi-task model outperformed the H1-hESC ${ }_{242}$ single-task model (Figure S3). In the latter case, the performance gap can be narrowed by changing the ${ }^{243}$ proportion of negative to positive training samples in the single-task framework, suggesting that any 244 additional gain granted by the multiple labels is eclipsed by the choice of negative sets. Nevertheless, ${ }^{245}$ ensembling single- and multi-task models together appears to be an effective method of improving predictive 246 performance, at least if antibodies and read lengths are kept consistent.

Another avenue we can explore for improving the model is hyperparameter tuning. We selected the 247 hyperparameters for the models in this work arbitrarily for demonstration and uniformity purposes (Table S1 S3. Although we have not yet implemented them, distributed computing hyperparameter tuning algorithms (Bergstra et al. 2013) can systematize hyperparameter selection and improve performance.

One of the chief criticisms of neural networks is that they are "black box" models. While neural networks can achieve great performances in predictive tasks, the exact reasons for why this is the case is not always entirely clear. In contrast to these criticisms, we can visualize and interpret aspects of the FactorNet model. By converting network kernels to motifs, we show that FactorNet can recover motifs that are known to contribute to binding (Figure 3A). DNase I footprint patterns help discriminate true binding sites from putative sites that simply match a motif. Previous TF binding prediction methods, such as Centipede, require users to supply motifs. FactorNet relaxes this strong assumption and essentially performs de novo motif discovery during the learning process to identify the sequence patterns that are most useful for binding prediction. Saliency maps can also help elucidate the complex regulatory grammar that govern TF binding by visualizing the spatial positions and orientations of multiple binding sites that work together to recruit TFs (Figure $3 \mathrm{D})$.

Our adherence to standardized file formats also makes FactorNet robust. For example, FactorNet can readily accept other genomic signals that were not included as part of the Challenge but are likely relevant to TF binding prediction, such as conservation and methylation. Along these same lines, if we were to refine our pre-processing strategies for the DNase-seq data, we can easily incorporate these improved features into our model as long as the data are available as bigWig files (Kent et al., 2010). Other sources of open chromatin information, such as ATAC-seq (Buenrostro et al., 2015) and FAIRE-seq (Giresi et al., 2007), can also be used to replace or complement the existing DNase-seq data. In addition, FactorNet is not necessarily limited to only TF binding predictions. If desired, users can provide the BED files of positive intervals to train predictive models for other markers, such as histone modifications. As more epigenetic datasets are constantly added to data repositories, FactorNet is already in a prime position to integrate both new and 
existing datasets.

In conclusion, FactorNet is a very flexible framework that lends itself to a variety of future research avenues. The techniques that we introduced in this paper will also be useful for the field of machine learning, 275 especially since neural network models are becoming increasingly popular in genomics. Some of the design ${ }_{276}$ elements of FactorNet were motivated by the specific properties inherent in the structure of the data. Many 277 of these properties are shared in data found in other applications of machine learning. For example, the ${ }^{278}$ directional nature and modularity of DNA sequences prompted us to search for a model that can discover ${ }^{279}$ local patterns and long-range interactions in sequences, which led us to ultimately select a hybrid neural ${ }_{280}$ network architecture that includes convolution and bidirectional recurrence. Natural language processing problems, such as topic modeling and sentiment analysis, can also benefit from such an architecture since language grammar is directional and modular. Another unique aspect of the data that guided our design is the double strandedness of DNA, which prompted us to adopt a Siamese architecture to handle pairs of input sequences (Figure 1). Protein-protein interaction prediction also involves sequence pairs and would likely serve as useful guidelines for other applications involving large imbalanced data, especially if recurrent models are utilized. We therefore expect that FactorNet will be of value to a wide variety of fields.

\section{Methods}

\section{ENCODE-DREAM Challenge dataset}

The ENCODE-DREAM Challenge dataset is comprised of DNase-seq, ChIP-seq, and RNA-seq data from the and pre-processing are based on hg19/GRCh37 release version of the human genome and GENCODE release 19 (Harrow et al. 2012). Data are restricted to chromosomes $\mathrm{X}$ and 1-22. Chromosomes 1, 8 and 21 are set aside exclusively for evaluation purposes and binding data were completely absent for these three genome is segmented into $200 \mathrm{bp}$ bins sliding every $50 \mathrm{bp}$. Each bin is labeled as bound (B), unbound (U) or ambiguously bound (A) depending on the majority label of all nucleotides in the bin. Ambiguous bins overlap peaks that fail to pass the IDR threshold of $5 \%$ and are excluded from evaluation. A more complete 


\section{Evaluation}

The TF binding prediction problem is evaluated as a two-class binary classification task. For each test

1. auROC. The area under the receiver operating characteristic curve is a common metric for evaluating classification models. It is equal to the probability that a classifier will rank a randomly chosen positive instance higher than a randomly chosen negative one.

2. auPR. The area under the precision-recall curve is more appropriate in the scenario of few relevant 308 items, as is the case with TF binding prediction (Quang and Xie, 2016). Unlike the auROC metric, the 309 auPR metric does not take into account the number of true negatives called.

3. Recall at fixed FDR. The recall at a fixed false discovery rate (FDR) represents a point on the precision-recall curve. Like the auPR metric, this metric is appropriate in the scenario of few relevant items. This metric is often used in applications such as fraud detection in which the goal may be to maximize the recall of true fraudsters while tolerating a given fraction of customers to falsely identify as fraudsters. The ENCODE-DREAM Challenge computes this metric for several FDR values.

As illustrated in Figure 1, the FactorNet Siamese architecture operates on both the forward and reverse 316 complement sequences to ensure that both strands return the same outputs during both training and ${ }_{317}$ prediction. Although a TF might only physically bind to one strand, this information cannot usually be inferred directly from the peak data. Thus, the same set of labels are assigned to both strands in the evaluation step.

\section{Features and data pre-processing}

FactorNet works directly with standard genomic file formats and requires relatively little pre-processing. 322 BED files provide the locations of reference TF binding sites and bigWig files (Kent et al. 2010$)$ provide ${ }^{323}$ dense, continuous data at single-nucleotide resolution. bigWig values are included as extra rows that are ${ }_{324}$ appended to the four-row one hot input DNA binary matrix. FactorNet can accept an arbitrary number of ${ }_{325}$ bigWig files as input features, and we found the following signals to be highly informative for prediction: ${ }_{326}$

1. DNase I cleavage. For each cell type, reads from all DNase-seq replicates were trimmed down to first ${ }_{327}$ nucleotide on the 5' end, pooled and normalized to 1x coverage using deepTools (Ramírez et al. 2014). ${ }_{328}$ 
2. 35 bp mapability uniqueness. This track quantifies the uniqueness of a 35 bp subsequence on the 329 positive strand starting at a particular base, which is important for distinguishing where in the genome 330 DNase I cuts can be detected. Scores are between 0 and 1, with 1 representing a completely unique 331 sequence and 0 representing a sequence that occurs more than 4 times in the genome. Otherwise, scores 332 between 0 and 1 indicate the inverse of the number of occurrences of that subsequence in the genome. It 333 is available from the UCSC genome browser under the table wgEncodeDukeMapabilityUniqueness35bp. $\quad 334$

In addition to sequential features, FactorNet also accepts non-sequential metadata features. At the cell 335 type level, we applied principal component analysis to the inverse hyperbolic sine transformed gene

\section{Training}

Our implementation is written in Python, utilizing the Keras 1.2.2 library (Chollet et al., 2015) with the 346 Theano 0.9.0 Bastien et al., 2012, Bergstra et al. 2010) backend. We used an NVIDIA Titan X Pascal GPU ${ }^{347}$ for training.

FactorNet supports single- and multi-task training. Both types of neural network models are trained using the Adam algorithm (Kingma and Ba, 2014) with a minibatch size of 100 to minimize the mean multi-task binary cross entropy loss function on the training set. We also include dropout (Srivastava et al. 2014 ) to reduce overfitting. One or more chromosomes are set aside as a validation set. Validation loss is evaluated at the end of each training epoch and the best model weights according to the validation loss are 
it is confidently bound to at least one TF. Bins that overlap a blacklisted region (ENCODE Project

\section{Single-task training}

Single-task training leverages data from multiple cell types by treating bins from all cell types as individually and identically distributed (i.i.d.) records. To make single-task training run efficiently, one bin is allotted per positive peak and these positive bins are included at most once per epoch for training. Each epoch, negative bins are also drawn randomly without replacement from the training chromosomes. For example, if we were to train on a single cell type that has 10,000 peaks for a particular TF, then we may train on 10,000 positive bins and 10,000 negative bins each epoch. Ambiguously bound bins are excluded from training.

FactorNet can only perform multi-task training when training on data from a single cell type due to the variation of available binding data for the cell types. For example, the ENCODE-DREAM Challenge provides reference binding data for 15 TFs for GM12878 and 16 TFs for HeLa-S3, but only 8 TFs are shared between the two cell types. Unlike the single-task models, which ignore ambiguous bins during training, the multi-task models assign negative labels to the ambiguous bins because of the frequent overlap of confidently and ambiguously bound regions. Compared to single-task training, multi-task training takes considerably longer to complete due to the larger number of positive bins. At the start of training, positive bins are identified by first segmenting the genome into 200 bins sliding every $50 \mathrm{bp}$ and discarding all bins that fail to overlap at least one confidently bound TF site. Each epoch, negative bins are drawn randomly with replacement from the training chromosomes.

\section{Bagging}

Ensembling is a common strategy for improving classification performance. At the time of the Challenge, we 380 implemented a simple ensembling strategy commonly called "bagging submissions", which involves averaging 381 predictions from two or more models. Instead of averaging prediction probabilities directly, we first convert 382 the scores to ranks, and then average these ranks. Rank averaging is more appropriate than direct averaging 383 if predictors are not evenly calibrated between 0 and 1, which is often the case with the FactorNet models. $\quad 384$ 


\section{Software availability}

Source code is available at the github repository http://github.com/uci-cbcl/FactorNet. In addition to the 386 source code, the github repository contains all models and data used for the ENCODE-DREAM Challenge. 387

\section{Acknowledgments}

We thank the ENCODE-DREAM challenge organizers for providing the opportunity to test and improve our $\quad 389$ method. We also thank David Knowles for helping with generating gene expression metadata features. 390

This work was supported by the National Institute of Biomedical Imaging and Bioengineering, National ${ }^{391}$ Research Service Award (EB009418) from the University of California, Irvine, Center for Complex Biological ${ }_{392}$ Systems and the National Science Foundation Graduate Research Fellowship under Grant No. 393 (DGE-1321846). Any opinion, findings, and conclusions or recommendations expressed in this material are 394 those of the authors and do not necessarily reflect the views of the National Science Foundation.

Conflict of interest statement.

None declared.

\section{References}

Alipanahi B, Delong A, Weirauch M T, and Frey B J. 2015. Predicting the sequence specificities of DNAand RNA-binding proteins by deep learning. Nat Biotechnol, 33(8):831-8. doi:10.1038/nbt.3300.

Angermueller C, Lee H J, Reik W, and Stegle O. 2017. DeepCpG: accurate prediction of single-cell DNA methylation states using deep learning. Genome biology, 18(1):67.

Bastien F, Lamblin P, Pascanu R, Bergstra J, Goodfellow I J, Bergeron A, Bouchard N, and Bengio Y. 2012. Theano: new features and speed improvements. Deep Learning and Unsupervised Feature Learning NIPS 2012 Workshop.

Bergstra J, Breuleux O, Bastien F, Lamblin P, Pascanu R, Desjardins G, Turian J, Warde-Farley D, and Bengio Y. 2010. Theano: a CPU and GPU math expression compiler. In Proceedings of the Python for scientific computing conference, volume 4, page 3. Austin, TX.

Bergstra J, Yamins D, and Cox D D. 2013. Making a Science of Model Search: Hyperparameter Optimization in Hundreds of Dimensions for Vision Architectures. ICML (1), 28:115-123. 
Boyle A P, Song L, Lee B K, London D, Keefe D, Birney E, Iyer V R, Crawford G E, and Furey T S. 2011. High-resolution genome-wide in vivo footprinting of diverse transcription factors in human cells. Genome research, 21(3):456-464.

Bromley J, Bentz J W, Bottou L, Guyon I, LeCun Y, Moore C, Säckinger E, and Shah R. 1993. Signature Verification Using A "Siamese" Time Delay Neural Network. IJPRAI, 7(4):669-688.

Buenrostro J D, Wu B, Chang H Y, and Greenleaf W J. 2015. ATAC-seq: A Method for Assaying Chromatin Accessibility Genome-Wide. Current protocols in molecular biology, pages 21-29.

Chen Y, Li Y, Narayan R, Subramanian A, and Xie X. 2016. Gene expression inference with deep learning. Bioinformatics. doi:10.1093/bioinformatics/btw074.

Chollet F et al. 2015. Keras. https://github.com/fchollet/keras.

Crawford, G et al. 2006. Genome-wide mapping of DNase hypersensitive sites using massively parallel signature sequencing (MPSS). Genome Res, 16(1):123-31. doi:10.1101/gr.4074106.

ENCODE Project Consortium. 2012. An integrated encyclopedia of DNA elements in the human genome. Nature, 489(7414):57-74. doi:10.1038/nature11247.

Ernst J and Kellis M. 2012. ChromHMM: automating chromatin-state discovery and characterization. Nature methods, 9(3):215-216.

Ernst J and Kellis M. 2015. Large-scale imputation of epigenomic datasets for systematic annotation of diverse human tissues. Nat Biotechnol, 33(4):364-76. doi:10.1038/nbt.3157.

Gardiner-Garden M and Frommer M. 1987. CpG islands in vertebrate genomes. Journal of molecular biology, 196(2):261-282.

Giresi P G, Kim J, McDaniell R M, Iyer V R, and Lieb J D. 2007. FAIRE (Formaldehyde-Assisted Isolation of Regulatory Elements) isolates active regulatory elements from human chromatin. Genome research, 17(6):877-885.

Graves A, Jaitly N, and Mohamed A R. 2013. Hybrid speech recognition with Deep Bidirectional LSTM. In Automatic Speech Recognition and Understanding, 2013 IEEE Workshop on, pages 273-278. doi:10.1109/ASRU.2013.6707742.

Graves A and Schmidhuber J. 2005. Framewise phoneme classification with bidirectional LSTM and other neural network architectures. Neural Networks, 18(5):602-610. 
Gupta S, Stamatoyannopoulos J A, Bailey T L, and Noble W S. 2007. Quantifying similarity between motifs. Genome Biol, 8(2):R24. doi:10.1186/gb-2007-8-2-r24.

Harrow J, Frankish A, Gonzalez J M, Tapanari E, Diekhans M, Kokocinski F, Aken B L, Barrell D, Zadissa

A, Searle S, et al. 2012. GENCODE: the reference human genome annotation for The ENCODE Project. Genome research, 22(9):1760-1774.

Hassanzadeh H R and Wang M D. 2016. DeeperBind: Enhancing prediction of sequence specificities of DNA binding proteins. In Bioinformatics and Biomedicine (BIBM), 2016 IEEE International Conference on, pages 178-183. IEEE.

Hesselberth J R, Chen X, Zhang Z, Sabo P J, Sandstrom R, Reynolds A P, Thurman R E, Neph S, Kuehn M S, Noble W S, Fields S, and Stamatoyannopoulos J A. 2009. Global mapping of protein-DNA interactions in vivo by digital genomic footprinting. Nat Methods, 6(4):283-9. doi:10.1038/nmeth.1313.

Hoffman M M, Buske O J, Wang J, Weng Z, Bilmes J A, and Noble W S. 2012. Unsupervised pattern discovery in human chromatin structure through genomic segmentation. Nature methods, 9(5):473-476.

John S, Sabo P J, Canfield T K, Lee K, Vong S, Weaver M, Wang H, Vierstra J, Reynolds A P, Thurman R E, et al. 2013. Genome-Scale Mapping of DNase I Hypersensitivity. Current protocols in molecular biology, pages 21-27.

Johnson D S, Mortazavi A, Myers R M, and Wold B. 2007. Genome-wide mapping of in vivo protein-DNA interactions. Science, 316(5830):1497-502. doi:10.1126/science.1141319.

Kelley D R, Snoek J, and Rinn J L. 2016. Basset: learning the regulatory code of the accessible genome with deep convolutional neural networks. Genome Res, 26(7):990-9. doi:10.1101/gr.200535.115.

Kent W J, Sugnet C W, Furey T S, Roskin K M, Pringle T H, Zahler A M, and Haussler D. 2002. The human genome browser at UCSC. Genome research, 12(6):996-1006.

Kent W J, Zweig A S, Barber G, Hinrichs A S, and Karolchik D. 2010. BigWig and BigBed: enabling browsing of large distributed datasets. Bioinformatics, 26(17):2204-2207.

Kharchenko P V, Tolstorukov M Y, and Park P J. 2008. Design and analysis of ChIP-seq experiments for DNA-binding proteins. Nature biotechnology, 26(12):1351-1359.

Kingma D and Ba J. 2014. Adam: A method for stochastic optimization. arXiv preprint arXiv:1412.6980. 
Leung M K K, Xiong H Y, Lee L J, and Frey B J. 2014. Deep learning of the tissue-regulated splicing code.

Bioinformatics, 30(12):i121-9. doi:10.1093/bioinformatics/btu277.

Mathelier, A et al. 2016. JASPAR 2016: a major expansion and update of the open-access database of transcription factor binding profiles. Nucleic Acids Research, 44(D1):D110-D115.

doi:10.1093/nar/gkv1176.

Medina-Rivera A, Defrance M, Sand O, Herrmann C, Castro-Mondragon J A, Delerce J, Jaeger S, Blanchet C, Vincens P, Caron C, Staines D M, Contreras-Moreira B, Artufel M, Charbonnier-Khamvongsa L, Hernandez C, Thieffry D, Thomas-Chollier M, and van Helden J. 2015. RSAT 2015: Regulatory Sequence Analysis Tools. Nucleic Acids Res, 43(W1):W50-6. doi:10.1093/nar/gkv362.

Mortazavi A, Pepke S, Jansen C, Marinov G K, Ernst J, Kellis M, Hardison R C, Myers R M, and Wold B J. 2013. Integrating and mining the chromatin landscape of cell-type specificity using self-organizing maps. Genome research, 23(12):2136-2148.

Mueller J and Thyagarajan A. 2016. Siamese Recurrent Architectures for Learning Sentence Similarity. In AAAI, pages 2786-2792.

Neph, S et al. 2012. An expansive human regulatory lexicon encoded in transcription factor footprints. Nature, 489(7414):83-90. doi:10.1038/nature11212.

Pique-Regi R, Degner J F, Pai A A, Gaffney D J, Gilad Y, and Pritchard J K. 2011. Accurate inference of transcription factor binding from DNA sequence and chromatin accessibility data. Genome Res, 21(3):447-55. doi:10.1101/gr.112623.110.

Qin Q and Feng J. 2017. Imputation for transcription factor binding predictions based on deep learning. PLoS computational biology, 13(2):e1005403.

Quang D, Chen Y, and Xie X. 2015a. DANN: a deep learning approach for annotating the pathogenicity of genetic variants. Bioinformatics, 31(5):761-3. doi:10.1093/bioinformatics/btu703.

Quang D and Xie X. 2014. EXTREME: an online EM algorithm for motif discovery. Bioinformatics, 30(12):1667-73. doi:10.1093/bioinformatics/btu093.

Quang D and Xie X. 2016. DanQ: a hybrid convolutional and recurrent deep neural network for quantifying the function of DNA sequences. Nucleic Acids Res, 44(11):e107. doi:10.1093/nar/gkw226. 
Quang D X, Erdos M R, Parker S C J, and Collins F S. 2015b. Motif signatures in stretch enhancers are enriched for disease-associated genetic variants. Epigenetics Chromatin, 8:23. doi:10.1186/s13072-015-0015-7.

Raj A, Shim H, Gilad Y, Pritchard J K, and Stephens M. 2015. msCentipede: Modeling Heterogeneity across Genomic Sites and Replicates Improves Accuracy in the Inference of Transcription Factor Binding. PLoS One, 10(9):e0138030. doi:10.1371/journal.pone.0138030.

Ramírez F, Dündar F, Diehl S, Grüning B A, and Manke T. 2014. deepTools: a flexible platform for exploring deep-sequencing data. Nucleic acids research, 42(W1):W187-W191.

Rhee H S and Pugh B F. 2011. Comprehensive genome-wide protein-DNA interactions detected at single-nucleotide resolution. Cell, 147(6):1408-1419.

Roadmap Epigenomics Consortium et al. 2015. Integrative analysis of 111 reference human epigenomes. Nature, 518(7539):317-30. doi:10.1038/nature14248.

Saito T and Rehmsmeier M. 2015. The precision-recall plot is more informative than the ROC plot when evaluating binary classifiers on imbalanced datasets. PloS one, 10(3):e0118432.

Sherwood R I, Hashimoto T, O'Donnell C W, Lewis S, Barkal A A, van Hoff J P, Karun V, Jaakkola T, and Gifford D K. 2014. Discovery of directional and nondirectional pioneer transcription factors by modeling DNase profile magnitude and shape. Nat Biotechnol, 32(2):171-8. doi:10.1038/nbt.2798.

Shrikumar A, Greenside P, and Kundaje A. 2017. Learning Important Features Through Propagating Activation Differences. arXiv preprint arXiv:1704.02685.

Simonyan K, Vedaldi A, and Zisserman A. 2013. Deep inside convolutional networks: Visualising image classification models and saliency maps. arXiv preprint arXiv:1312.6034.

Srivastava N, Hinton G, Krizhevsky A, Sutskever I, and Salakhutdinov R. 2014. Dropout: A simple way to prevent neural networks from overfitting. The Journal of Machine Learning Research, 15(1):1929-1958.

Sundermeyer M, Alkhouli T, Wuebker J, and Ney H. 2014. Translation modeling with bidirectional recurrent neural networks. In Proceedings of the Conference on Empirical Methods on Natural Language Processing, October.

Thorvaldsdóttir H, Robinson J T, and Mesirov J P. 2013. Integrative Genomics Viewer (IGV):

high-performance genomics data visualization and exploration. Briefings in bioinformatics, 14(2):178-192. 

aCC-BY 4.0 International license.

Zhou J and Troyanskaya O G. 2015. Predicting effects of noncoding variants with deep learning-based sequence model. Nat Methods, 12(10):931-4. doi:10.1038/nmeth.3547.

Zhu W, Lan C, Xing J, Li Y, Shen L, Zeng W, and Xie X. 2016. Co-occurrence Feature Learning for Skeleton based Action Recognition using Regularized Deep LSTM Networks. The 30th AAAI Conference on Artificial Intelligence (AAAI-16). 


\section{Supporting Information}

Table S1. Summary and description of the hyperparameters used for the single-task models in Figure S2B.

\begin{tabular}{|c|c|c|}
\hline Hyperparameter & Value & Description \\
\hline -v validchroms & $\begin{array}{l}\text { chr3 chr5 chr7 chr10 chr12 chr14 } \\
\text { chr16 chr18 chr20 chrX }\end{array}$ & $\begin{array}{l}\text { Sequences on these chromosomes are set } \\
\text { aside for validation. }\end{array}$ \\
\hline -e epochs & $\begin{array}{l}200 \text { (ZNF143, TAF1), } 300 \text { (E2F1, } \\
\text { GABPA) }\end{array}$ & $\begin{array}{l}\text { Max number of epochs to train before } \\
\text { training ends. }\end{array}$ \\
\hline -ep patience & $\begin{array}{l}200 \text { (ZNF143, TAF1), } 300 \text { (E2F1, } \\
\text { GABPA) }\end{array}$ & $\begin{array}{l}\text { Number of epochs with no improvement } \\
\text { in the validation loss. }\end{array}$ \\
\hline -lr learningrate & 0.00001 & $\begin{array}{l}\text { Learning rate for the Adam optimizer. We } \\
\text { decreased it from the default value of } 0.001 \\
\text { to smooth the learning curves. }\end{array}$ \\
\hline -n negatives & 1 & $\begin{array}{l}\text { Number of negative bins to sample per } \\
\text { positive bin per epoch. }\end{array}$ \\
\hline -L seqlen & 1000 & $\begin{array}{l}\text { Length, in bps, of input sequences to the } \\
\text { model. }\end{array}$ \\
\hline -w motifwidth & 26 & $\begin{array}{l}\text { Width, in bps, of the convolutional ker- } \\
\text { nels. }\end{array}$ \\
\hline -k kernels & 32 & Number of kernels/motifs in the model. \\
\hline -r recurrent & 32 & $\begin{array}{l}\text { Number of recurrent units (in one direc- } \\
\text { tion) in the model. }\end{array}$ \\
\hline -d dense & 128 & $\begin{array}{l}\text { Number of units in the dense layer in the } \\
\text { model. }\end{array}$ \\
\hline -p dropout & 0.5 & $\begin{array}{l}\text { Dropout rate between the recurrent and } \\
\text { dense layers. Also the dropout rate be- } \\
\text { tween the dense and sigmoid layers. }\end{array}$ \\
\hline -m metaflag & False & $\begin{array}{l}\text { Flag for including cell type-specific meta- } \\
\text { data features (usually gene expression). }\end{array}$ \\
\hline -g gencodeflag & True & $\begin{array}{l}\text { Flag for including } \mathrm{CpG} \text { island and gene } \\
\text { annotations. }\end{array}$ \\
\hline -mo motifflag & True & $\begin{array}{l}\text { Flag for initializing two of the kernels to } \\
\text { the PWM of the canonical motif (forward } \\
\text { and reverse complement). }\end{array}$ \\
\hline -s randomseed & Varies & Random seed for reproducibility. \\
\hline
\end{tabular}


Table S2. Hyperparameters used for the multi-task models in Figures 3 and $\mathbf{S 2}$ S3. Unspecified values should be assumed to be the same as those found in Table S1.

\begin{tabular}{|c|c|c|}
\hline Hyperparameter & Value & Notes \\
\hline -v validchroms & chr11 & \\
\hline -e epochs & 20 & $\begin{array}{l}\text { Fewer epochs needed for multi-task train- } \\
\text { ing due to the large number of training } \\
\text { bins. }\end{array}$ \\
\hline -ep patience & 20 & \\
\hline -lr learningrate & 0.001 & $\begin{array}{l}\text { Default value of } 0.001 \text { is sufficient for most } \\
\text { applications. }\end{array}$ \\
\hline -n negatives & 1 & \\
\hline -g gencodeflag & False & $\begin{array}{l}\text { Multi-task training does not currently in- } \\
\text { corporate any metadata features. }\end{array}$ \\
\hline
\end{tabular}

Table S3. Hyperparameters used for the single-task models in Figures S2 C-D and S3. Unspecified values should be assumed to be the same as those found in Table S1.

\begin{tabular}{lll} 
Hyperparameter & Value & Notes \\
\hline -v validchroms & chr11 & $\begin{array}{l}\text { Need more epochs than multi-task train- } \\
\text { ing due to fewer positive bins. }\end{array}$ \\
-e epochs & 100 & $\begin{array}{l}\text { Default value of } 0.001 \text { is sufficient for most } \\
\text { applications. }\end{array}$ \\
-ep patience & 20 & $\begin{array}{l}\text { In some cases, increasing this value from } \\
\text {-lr learningrate }\end{array}$ \\
-n negatives & 0.001 & Varies (but usually 1) \\
single-task models.
\end{tabular}




\section{GENCODE v19}

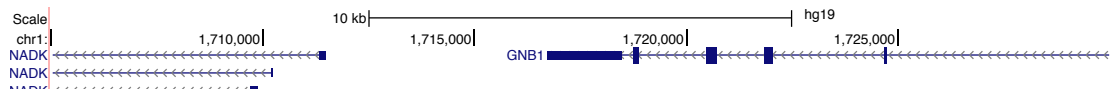

ChIP-seq fold change signal

FactorNet predictions

Peaks

ChIP-seq fold change signal

FactorNet predictions

Peaks

ChIP-seq fold change signal

FactorNet predictions

Peaks

ChIP-seq fold change signal

FactorNet predictions

Peaks

NADK

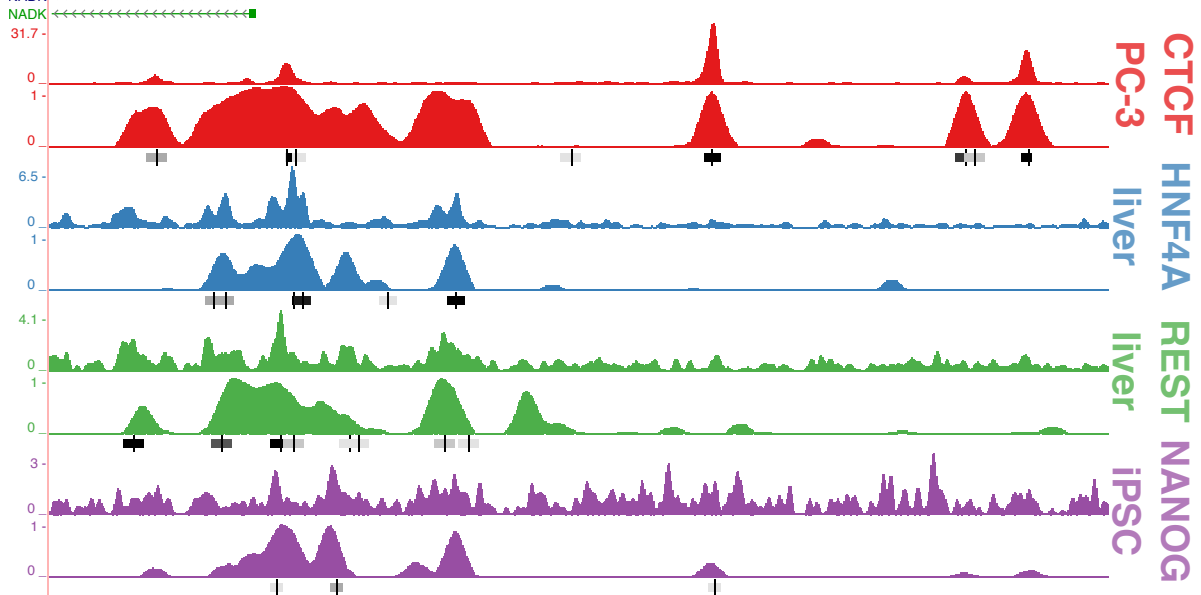

Figure S1. FactorNet cross-cell type predictions are comparable to ChIP-seq signals and peaks.

A genome browser shot similar to Figure 2 A focusing on the NADK/GNB1 locus. 


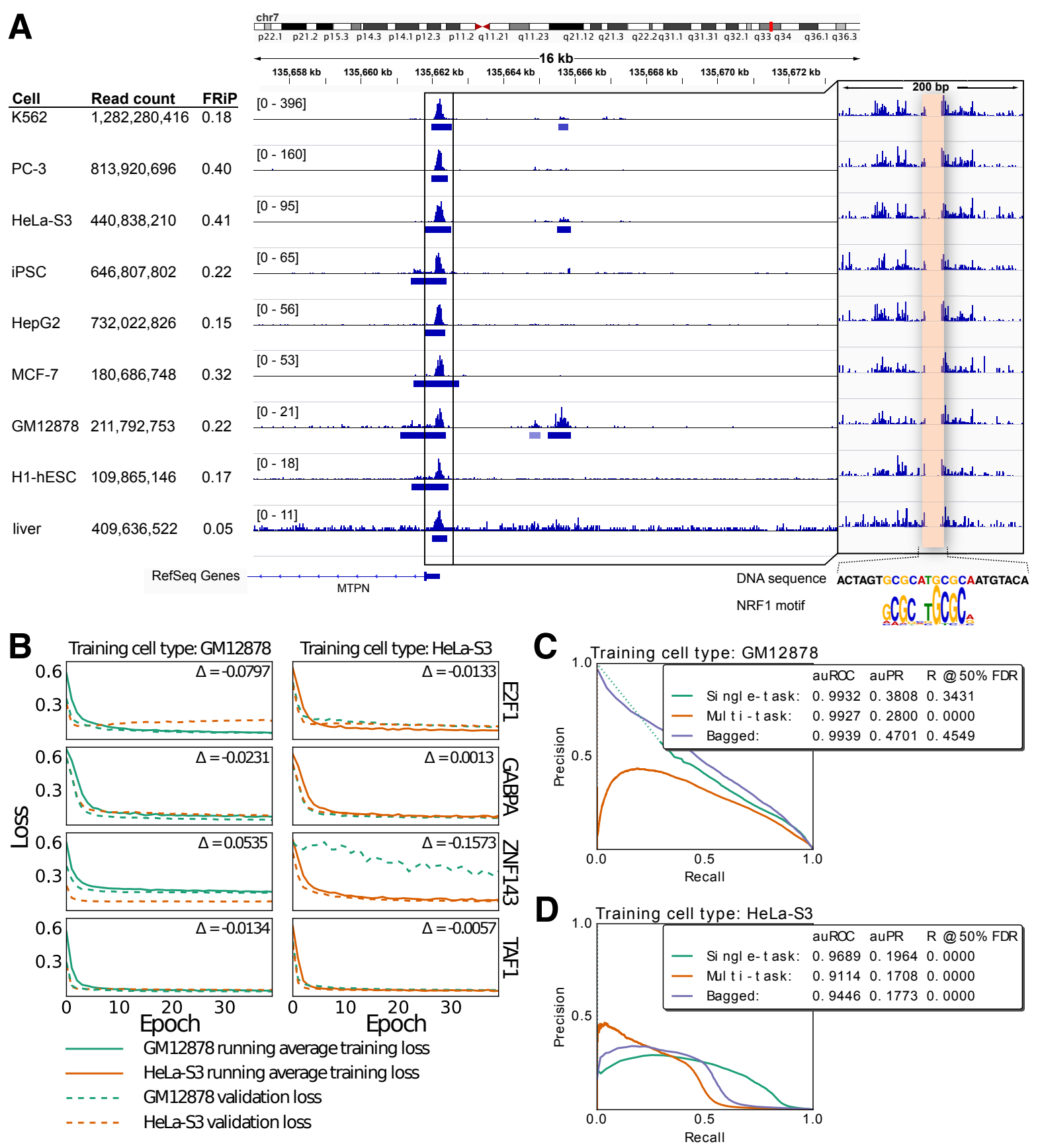

Figure S2. Variation in cell type-specific datasets influence cross-cell type predictive performance. (A) IGV (Thorvaldsdóttir et al., 2013) browser screenshot displays pooled DNase I cleavage signal and conservative DNase-seq peaks for eight cell types. The inset is a magnified view at the MTPN promoter, a known NRF1 binding site. (B) Each plot displays learning curves of single-task models trained on either GM12878 or HeLa-S3. We generated within- and cross-cell type validation sets by extracting an equal number of positive and negative bins from the validation chromosomes. The difference between the smallest withinand cross-cell type validation losses are displayed in each plot. ( $C$ and $D$ ) Precision-recall curves of singleand multi-task models evaluated on the E2F1/K562 testing set trained exclusively on either GM12878 or HeLa-s3. Dotted lines indicate points of discontinuity. Model weights were selected based on the within-cell type validation loss on chr11. We generated single-task scores by bagging scores from two single-task models initialized differently. Final bagged models ensemble respective single- and multi-task models. 


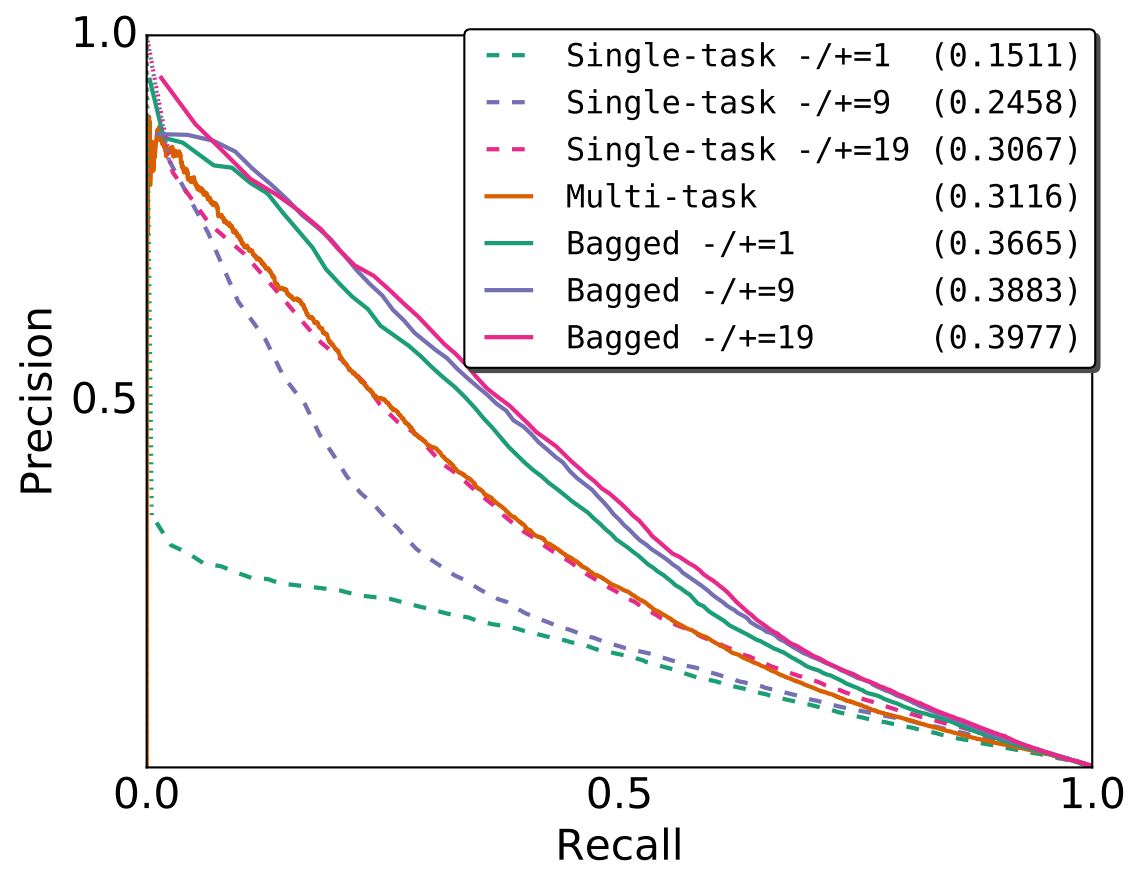

Figure S3. Comparison of single- and multi-task training. Cross-cell type precision-recall curves of single-task and multi-task NANOG binding prediction models trained on H1-hESC and evaluated on iPSC. Model weights were selected based on the within-cell type validation loss on chr11. We generated single-task scores by bagging scores from two single-task models initialized differently. The three single-task models differ in the ratio of negative-to-positive bins per training epoch. The bagged models are the rank average scores from the multi-task model and one of the three single-task models. auPR scores are in parentheses. Both training and testing ChiP-seq datasets use the ENCAB000AIX antibody. 